\title{
Mushroom Poisoning in North America
}

\author{
Michael W. Beug
}

The Evergreen State College, 2700 Evergreen Parkway NW, Olympia, WA 98505, USA

Americans remain a very mycophobic people, although in the last 30 years popular mushroom identification field guides, a journal for amateurs (Mushroom: The Journal), and more than 64 mushroom clubs have led to a growing number of mushroom enthusiasts. In the Northwest, commercial mushroom picking has become economically important, with many pickers out gathering in the wild, sometimes with little or no knowledge of mushroom identification or mushroom ecology. All of this occurs with relatively few cases of mushroom poisoning, although a parent finding a child with half a mushroom dangling from his mouth will inevitably lead to panic.

In order to assist doctors, who are often unfamiliar with mushroom poisoning, and to better inform the public, the North American Mycological Association (NAMA) maintains a continent-wide group of identifiers who stand ready to assist doctors and poison centers in identifying mushrooms suspected of causing poisoning. NAMA provides educational materials on mushroom identification and information on diagnosis and treatment of mushroom poisoning and promotes the reporting of mushroom poisoning and the treatment used.

The challenge we face is to get a more complete reporting of poisonous incidences in order to un- derstand the frequency and severity of mushroom poisoning in North America. New patient confidentiality rules also inhibit reporting of poisonings, even though the reports do not involve identifying individuals involved.

What is clear is that the majority of cases reported to poison centers involve ingestion of mushrooms by children where there are no adverse symptoms. Cases involving symptoms typically involve gastro-intestinal distress. The distress is at times severe and may leave the individual in a weakened state for a week or more. The main threat of death from mushroom ingestion is from highly toxic species by young children and by individuals not otherwise in good health. Adults in good health normally survive ingestion of even the deadliest mushrooms provided they get prompt, good medical treatment and provided that they have not consumed a huge amount of the toxic species.

A major challenge in North America is to educate recent immigrants who may make mushrooms a significant part of their diet and may pick toxic species that resemble edible species from their home country.

This presentation will focus on characteristics of mushrooms in North America that cause substantial numbers of poisoning, with an emphasis on cases in the past 4 years. 